\title{
SUSTENTABILIDAD DE LA PRODUCCIÓN CAPRINA EN LA MESETA CENTRAL DE MENDOZA, ARGENTINA
}

\section{SUSTAINABILITY OF CAPRINE PRODUCTION ON THE CENTRAL PLATEAU OF MENDOZA, ARGENTINA}

\author{
Patricio Dayenoff ${ }^{1 *}$ y Javier Macario ${ }^{1}$ \\ Fecha de recepción: 14 de dicembre de 2016 \\ Fecha de aceptación: 20 de marzo de 2017
}

\begin{abstract}
Resumen - El objetivo del presente trabajo fue conocer la composición botánica de la ingesta caprina en pastizal natural entre los años 20112013 y la calidad forrajera de las especies que mayor participación presentaron a lo largo de los ciclos vegetativos comprendidos entre 2013-2015, en la Meseta Central de Mendoza. Para ello se evaluó por micro-histología de heces de 54 cabras adultas tipo criollo en las fases Rebrote, Floración y Reposo vegetativo. La calidad forrajera de las especies con mayor participación se analizó por niveles de proteína bruta (AOAC, 1980), fibra detergente neutro, fibra detergente ácido (Van Soest et al., 1991) y digestibilidad in vitro materia seca (Barnes y Marten, 1980). Las medias y desvíos estándar se estudiaron por ANAVA y test de Tukey. Los arbustos fueron las especies más consumidas, 53,03\% en Rebrote; $64,7 \%$ en Floración, y $74,2 \%$ en Latencia, destacando las especies Lycium sp., Schinus sp. y Prosopis sp, como las que participaron en los tres momentos fenológicos del pastizal natural, con niveles de ingesta superior a $8 \%$ en cada momento.
\end{abstract}

Palabras clave:

Caprinos, pastizal natural, ingesta, calidad forrajera.
Abstract - The objective of the present work was know the botanical composition of the goat's intake in natural pasture, between them years 2011-2013, and the quality forage of them species that greater participation presented along them cycles vegetative covered between 2013-2015, in the Plateau Central of Mendoza. This was evaluated in 54 Criollo adult goats by Fecal Micro-histology method in Regrowth, Flowering and vegetative Latency phases. Forage quality of species with greater participation was assessed by levels of crude protein, Official Methods of Analices (AOAC, 1980), Neutral Detergent Fiber, Acid Detergent Fiber (Van Soest, Robertson and Lewis, 1991) and Digestibility In Vitro Dry Matter (Barnes and Marten, 1980). The media and standard desviation was studied by ANAVA and Tukey test. The bushes were the most consumed species, 53,03\% on Regrowth, $64.7 \%$, in Florewing and $74.2 \%$ in Latency, highlighting species Lycium sp., Schinus sp. and Prosopis sp, as which participated in the three phenological moments of the natural pasture, with levels of intake upper to the $8 \%$ in, each moment.

\section{Keywords:}

Goats, natural pasture, intake, forage quality. 


\section{Introducción}

E uso racional del pastizal natural, a través de la carga animal, es factor determinante para logar una administración sustentable de los recursos forrajeros, más aún en las zonas áridas donde el equilibrio ambiental es muy frágil y una presión de pastoreo inadecuada provoca desorden y pérdida de biodiversidad tanto vegetal como animal, que deriva en una desertificación por efecto diente, muchas veces difícil de revertir (Laycock, 1994).

Asimismo, Scarnecchia (1994) recomienda que, al introducir caprinos en una superficie para un aprovechamiento ganadero, se tenga en cuenta la dinámica de las variables participantes en el sistema, como el crecimiento vegetal, su desaparición al poner ahí al consumidor, los niveles de carga ganadera y la especie animal, entre otras.

Por otra parte, una carga ganadera racional estimula la producción forrajera por acumulación de reservas de las plantas, mientras que una inadecuada, así como la mala elección de la época de utilización del rebrote, provoca agotamiento de las reservas vegetales, dañando seriamente la regeneración del forraje (Radclife, 1985). Hall, Specht y Early (1964) sostienen que la sensibilidad de las diferentes especies vegetales de las zonas áridas a la presión de pastoreo depende de su palatabilidad y de su susceptibilidad al daño por defoliación.

La vegetación arbustiva es el estrato predominante en la región de la meseta central del sur de Mendoza, zona árida templada que abarca una superficie de algo más de 1,5 millones de hectáreas (Dayenoff, Macario y Moreno, 2013), cuya principal actividad comercial es la producción caprina extensiva y el producto es el cabrito lechal.

Ese estrato vegetal es el que mayor aporte alimenticio provee para la alimentación del ganado caprino (López-Trujillo y García Elizondo, 1995), estrato que se aprovecha en distintas proporciones durante los diferentes momento fenológicos del ciclo vegetativo de los arbustos (Dayenoff, Bolaño, Aguirre y
Giovanardi, 1999), (Omphile, Aganga y Malamba, 2003) (Brinkmann, Patzelt, Dickhoefer, Schlecht y Buerkert, 2009) y que en distintos trabajos de investigación demostraron que normalmente no existen especies vegetales de preferencia constante, encontrando que los caprinos muestran una alta selectividad, al elegir distintas plantas y partes de ellas a lo largo del año (Dayenoff, 1998; Sanon et al., 2007; Glasser, Landau, Ungar, Perevolotsky, Dvash, Muklada, Kababya y Walker, 2012).

La predisposición del caprino al consumo de arbustos (Gihad, El-Gallal, Allan y El-Badawy, 1984) estaría influenciada por varios factores, siendo uno de los más destacables la mejor calidad forrajera que éstos ofrecen frente a las gramíneas en el mismo momento fenológico (Papachristou y Paranastis, 1994), situación referenciada por Siebert, Newman y Nelson (1968), quienes postulan que el contenido proteico de los vegetales es el parámetro más adecuado para evaluar, a posteriori, el efecto de los animales sobre la vegetación.

Para iniciar el proceso de manejo sustentable de la vegetación natural como base alimenticia y antes de realizar el ajuste de la capacidad a fin de sustentar caprinos de una superficie, es necesario conocer previamente las especies vegetales que existen, su participación en la ingesta caprina y el aporte de nutrientes de dichas especies en los diferente momentos fenológicos del pastizal natural (Severson y Debano, 1991) y realizar una presión de pastoreo animal adecuado, sin sobreestimar la vegetación total existente (Dayenoff, 1998).

El objetivo de este trabajo fue evaluar la ingesta caprina en pastoreo directo del pastizal natural del área de la Meseta Central de Mendoza y la calidad forrajera de las especies más consumidas por el ganado caprino en los tres momentos fenológicos.

\section{Material y método}

El trabajo se desarrolló en la región de la Meseta Central de Mendoza, zona que posee una superficie 
superior a 1,5 millones de hectáreas, ubicada geográficamente entre los $68^{\circ} 26^{\prime} y$ los $69^{\circ} 30^{\prime}$ de longitud oeste y entre los $34^{\circ} 54^{\prime}$ y los $35^{\circ} 54^{\prime}$ de latitud sur, aproximadamente, con una altitud promedio de 1760 metros sobre el nivel del mar.

La región está comprendida dentro de un área de clima árido templado continental, con inviernos muy fríos cuya temperatura mínima llega a los $-16^{\circ}$ centígrados y veranos con máximas que alcanzan los $38^{\circ}$ centígrados; las precipitaciones son de tipo primavera-estivales, muy oscilantes y erráticas, y el promedio histórico es de 154 mm/año.

La Meseta se ubica al este de la Cordillera de Los Andes, a 80 kilómetros en línea recta, posee un suelo pedregoso y franco-arenoso, no tiene curso de agua permanente y su expresión es temporal y ligada a la época de precipitaciones. Como referencia geográfica, se localiza a 140 kilómetros al suroeste de la ciudad de San Rafael y a 120 kilómetros al noreste de Malargüe, en la provincia de Mendoza, Argentina.

Desde el punto de vista fitogeográfico, es una zona de transición entre Patagonia, la provincia de Monte y la del Espinal; las especies arbustivas presentes en las comunidades vegetales son de los géneros Lycium, Chuquiraga, Prosopis, Ephedra, Gutierrezia, Verbena y Baccharis, a las que se puede agregar Monthea aphylla, Bouganvillaea spinosa, Schinus polygamus, Condalia microphyla, Verbena alatocarpa y Bredemeyera microphylla. Atriplex lampa.

El estrato de sub-arbustos está formado por Cassia aphylla, Acantholyppia seriphioides, Perezia recurvata, Baccharis darwini, entre otras, y dentro de las gramíneas más frecuentes se encuentran Stipa tenuis, Stipa speciosa, Stipa neaei, Poa ligularis, Poa. Lanuginosa, Schismus barbatus, Bromus tectorum, Digitaria sp, Panicum sp y Vulpia sp (Cabrera, 1976).

Para la toma de muestras se seleccionaron tres establecimientos comerciales de la zona, donde se explota ganado caprino de manera tradicional, teniendo cada uno 680 cabras aproximadamente, con alimentación basada en pastoreo diurno libre y encierre nocturno, sin suplementación ni ajuste de carga animal, con escasa aplicación de tecnología de base científica; como característica diferencial, los productores locales realizan veranada, por lo que los animales utilizan el pastizal natural del área como fuente de alimentación en forma permanente entre los meses de marzo-diciembre, teniendo un descanso relativo de uso en el resto del año.

Cabe señalar que en la Meseta también pastorean ovinos, bovinos, equinos y una diversidad de fauna silvestre como conejos, guanacos, mulitas y suris, entre otros. Ahí no hay alambrados de separación entre las propiedades, por lo que los animales pastan libremente, recorriendo en ocasiones hasta 20 kilómetros de distancia desde sus establecimientos buscando alimentarse, situación más marcada en periodos de sequías.

En cada establecimiento se seleccionaron 18 cabras adultas, tipo criollo regional, las que se identificaron con caravanas plásticas en el pabellón auricular izquierdo, tomado una muestra de heces directamente del recto de los animales en horario matutino, previo a la salida al pastoreo, de 50 gramos por ejemplar, aproximadamente.

Los muestreos se realizaron en tres estaciones vegetativas del pastizal natural: Rebrote (octubrenoviembre, inicio de estación húmeda), Floración (enero, durante la estación húmeda) y Latencia vegetativa (agosto, estación seca), durante los años 2011, 2012 y 2013.

Las muestras se secaron en estufa a $62^{\circ} \mathrm{C}$ hasta peso constante y se enviaron a laboratorio para determinar su composición botánica por el método de micro-histología de heces (Holechek, 1982).

Para el estudio de calidad forrajera se eligieron las tres especies arbustivas de mayor participación en la ingesta, considerando que deberían aparecer como mínimo en dos periodos y con un nivel de consumo superior a 5\% del total de la ingesta, valor que indica alta preferencia (Taylor, Kothmann, Merrill y Elledge, 1980).

Las muestras de material vegetal de referencia se tomaron en los ciclos vegetativos 2013-2014 y 20142015, y en los mismos momentos que para el control 
de ingesta, tomando muestras de hojas, tallos de hasta tres milímetros y flores de las tres especies arbustivas seleccionadas (Schinus sp., Lycium sp. y Prosopis sp), recolectando 10 muestras de cada especie, en cada momento vegetativo y en cada año de muestreo.

El material se secó en estufa a $62^{\circ} \mathrm{C}$ hasta obtener materia seca, se colocó nuevamente en bolsas de papel madera, identificando por separado cada una y se envió a laboratorio para su análisis. El material se examinó por el método AOAC (1980) para determinar el contenido de proteína bruta y se sometió al método de Van Soest, Robertson y Lewis (1991), para el estudio y medición de los niveles de fibra detergente neutro y fibra detergente ácido.

Para el cálculo de digestibilidad de la materia seca se empleó la fórmula porcentaje de digestibilidad in vitro de la materia seca $=88.9(0.779 \times$ \% Fibra Detergente Ácido) (Barnes y Marten, 1980).
El diseño experimental respondió a un modelo completamente aleatorizado, las diferencias de medias entre especies que se repiten en distintos momentos se estudiaron por análisis de varianza, aplicando el test de Tukey por medio de paquete informático Infostat 2.0.

\section{Resultados}

Se resalta que en la Tabla 1 figuran únicamente las especies vegetales que las cabras seleccionaron en su ingesta en más de $1 \%$ del total consumido en los diferentes momentos del año. Se advierte que las cabras que pastorean en la Meseta Central de Mendoza utilizaron una cantidad similar de especies vegetales en los tres momentos vegetativos, 11 especies durante la fase de Rebrote, 11 en el momento de Floración y 10 durante el Reposo vegetativo.
Tabla 1.

Distribución porcentual de la ingesta caprina en la Meseta Central de Mendoza, según especie y momento vegetativo

Letras diferentes, mismo renglón, diferencia estadística significativa $(p \leq 0,05)$.

\begin{tabular}{|c|c|c|c|}
\hline GÉNERO & REBROTE & FLORACIÓN & LATENCIA \\
\hline Poa & $9,8 \pm 4,12 \mathrm{a}$ & $1,33 \pm 1,63 \mathrm{~b}$ & $1,2 \pm 4,67 \mathrm{~b}$ \\
\hline Lycium & $14,22 \pm 2,68 \mathrm{a}$ & $15,67 \pm 3,88 \mathrm{~b}$ & $14,38 \pm 5,32 \mathrm{~b}$ \\
\hline Senecio & $4,21 \pm 5,66 \mathrm{a}$ & $14,67 \pm 5,16 \mathrm{~b}$ & $14,10 \pm 11,16 \mathrm{~b}$ \\
\hline Stipa & $5,64 \pm 5,37 \mathrm{a}$ & $4,08 \pm 3,79 \mathrm{a}$ & $3,21 \pm 3,65 \mathrm{a}$ \\
\hline Schinus & $8,42 \pm 3,58 \mathrm{a}$ & $12,54 \pm 4,32 \mathrm{~b}$ & $18,43 \pm 8,07 \mathrm{c}$ \\
\hline Prosopis & $18,64 \pm 4,94 \mathrm{a}$ & $15,33 \pm 4,50 \mathrm{~b}$ & $12,38 \pm 2,16 \mathrm{c}$ \\
\hline Jumelia & $6,08 \pm 5,67 \mathrm{a}$ & $10,5 \pm 4,03 \mathrm{~b}$ & $14,7 \pm 5,27 \mathrm{c}$ \\
\hline Setaria & $2,15 \pm 2,83$ & & \\
\hline Digitaria & $7,64 \pm 6,07$ & & \\
\hline Panicum & $10,8 \pm 9,12$ & & \\
\hline Verbena sp & $1,61 \pm 1,79$ & & \\
\hline Pappophorum & & $3,10 \pm 3,07$ & \\
\hline Panicum & & $2,04 \pm 3,95$ & \\
\hline Hyalis & & $4,33 \pm 6,28$ & \\
\hline Gomphrena & & $7,67 \pm 1,63$ & \\
\hline Bromus & & & $4,60 \pm 3,41$ \\
\hline Hordeum & & & $1,02 \pm 1,05$ \\
\hline Fabiana & & & \\
\hline
\end{tabular}


A su vez, se encontró que el estrato arbustivo ha sido el más ingerido por las cabras, teniendo en la fase de Rebrote una participación de 53,03\% de la ingesta caprina, contra 35,24\% de presencia del estrato herbáceo.

Asimismo, durante la Floración, los arbustos representaron $64,7 \%$ de lo ingerido, mientras que las gramíneas sólo fueron $14,64 \%$ y finalmente, durante la Latencia vegetativa, las arbustivas fueron ingeridas $74,2 \%$ y las gramíneas únicamente $14,6 \%$.

Una situación similar se presenta al considerar el consumo por especie, la tabla muestra que una especie vegetal del estrato arbustivo fue la más consumida: Prosopis sp., en el Rebrote, con una participación de $18,54 \pm 4,94 \%$ de la ingesta; Lycium sp., en la Floración, con $15,67 \pm 3,88 \%$, y Schinus $s p$, durante la Latencia vegetativa, con $18,43 \pm 8,07 \%$.
También en la tabla 1 se observa que sólo siete especies vegetales aparecen en los tres momentos evaluados y dentro de éstas, los arbustos son las predominantes, con $71,44 \%$.

Por otra parte, analizando las especies vegetales que participaron en los tres momentos, se ve una variación estadística significativa $(p \leq 0,05)$ en cuanto a la selección de las cabras respecto de cada una de ellas, encontrando que ninguna especie predominó en los tres momentos de muestreo.

En cuanto a la calidad forrajera de las tres especies seleccionadas, la tabla 2 muestra que para un mismo periodo vegetativo no se encontraron diferencias estadísticas significativas en cada una de las variables analizadas.

Tabla 2.

Media y desvío estándar de los valores de proteína bruta (PB), fibra detergente neutro (FDN) y fibra detergente ácido (FDA), según especie vegetal y periodo vegetativo

\begin{tabular}{|c|c|c|c|c|}
\hline PERIODO & & REBROTE & FLORACIÓN & LATENCIA \\
\hline Latencia & Lycium & $9,9 \pm 0,7 a$ & $53,9 \pm 2,3 a$ & $31,7 \pm 1,5 a$ \\
\hline & Schimus & $9,1 \pm 0,9 a$ & $54,5 \pm 1,5 a$ & $34,1 \pm 3,3 a$ \\
\hline & Prosopis & $8,3 \pm 0,7 a$ & $57,4 \pm 2,5 a$ & $36,9 \pm 2,1 a$ \\
\hline Rebrote & Lycium & $20,5 \pm 2,1 a$ & $38,7 \pm 2,6 a$ & $25,6 \pm 2,3 a$ \\
\hline & Schimus & $19,9 \pm 1,1 a$ & $34,6 \pm 2,2 a$ & $25,1 \pm 1,5 a$ \\
\hline & Prosopis & $19,1 \pm 1,3 a$ & $35,1 \pm 2,1 a$ & $25,2 \pm 1,3 a$ \\
\hline Floración & Lycium & $13,9 \pm 0,8 a$ & $47,1 \pm 3,2 a$ & $30,4 \pm 2,1 a$ \\
\hline & Schimus & $14,2 \pm 1,4 a$ & $49,2 \pm 1,8 a$ & $30,3 \pm 2,5 a$ \\
\hline & Prosopis & $13,6 \pm 0,5 a$ & $49,9 \pm 3,1 a$ & $31,8 \pm 2,8$ a \\
\hline
\end{tabular}

Letras distintas misma columna, según periodo $(p \leq 0,01)$.
A través del análisis de cada especie vegetal en particular, Lycium sp. mostró niveles más altos es su calidad forrajera en el lapso de Rebrote, momento en que los valores fueron: proteína bruta $20,5 \pm 2,1 \%$, fibra detergente neutro 38,7 \pm 2.6 ,

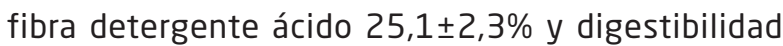
in vitro materia seca de $69,4 \pm 0,3 \%$, mostrando una diferencia estadística significativa $(p \leq 0,01)$ con los periodos de Latencia y Floración; a su vez, los niveles de fibra detergente ácido y digestibilidad in vitro materia seca presentaron diferencia estadística significativa $(p \leq 0,01)$ en el Rebrote, siendo similares entre Latencia y Floración, como se presenta en la tabla 3. 
Tabla 3.

Media y desvío estándar de los valores de proteína bruta, fibra detergente neutro, fibra detergente ácido y digestibilidad in vitro materia seca de Lycium sp., en distintos periodos vegetativos (2013-2014)

\begin{tabular}{|c|c|c|c|c|}
\hline LYCIUMSP. & $\% \mathrm{~PB}$ & $\% \mathrm{FDN}$ & $\% \mathrm{FDA}$ & $\%$ DMO \\
\hline Latencia & $9,9 \pm 0,7^{\mathrm{a}}$ & $53,9 \pm 2,3^{\mathrm{a}}$ & $31,7 \pm 1,5 \mathrm{~b}$ & $64,2 \pm 1,7 \mathrm{a}$ \\
\hline Rebrote & $20,5 \pm 2,1 \mathrm{~b}$ & $38,7 \pm 2,6 \mathrm{~b}$ & $25,1 \pm 2,3 \mathrm{a}$ & $69,4 \pm 0,3 \mathrm{~b}$ \\
\hline Floración & $13,9 \pm 0,8 \mathrm{c}$ & $47,1 \pm 3,2 \mathrm{c}$ & $30,4 \pm 2,2 \mathrm{~b}$ & $65,8 \pm 2,1 \mathrm{a}$ \\
\hline
\end{tabular}

Letras distintas misma columna diferencia estadística significativa $(p \leq 0,01)$.
Esa misma circunstancia se observó en el periodo vegetativo 2014-2015 que se muestra en la tabla 4 , en la cual se ve que tanto los valores de proteína bruta como los de fibra detergente neutro mostraron variaciones estadísticas significativas $(p \leq 0,01)$ para los tres momentos vegetativos; mientras que los niveles de fibra detergente ácido, así como los de digestibilidad in vitro materia seca se mostraron similares y con diferencia estadística significativa $(p \leq 0,01)$ en la fase de Rebrote.

\begin{tabular}{|c|c|c|c|c|}
\hline LYCIUM & $\% \mathrm{~PB}$ & $\%$ FDN & $\%$ FDA & $\%$ DMO \\
\hline Latencia & $10,2 \pm 1,3 \mathrm{a}$ & $50,4 \pm 2,1 \mathrm{a}$ & $29,6 \pm 1,1 \mathrm{~b}$ & $64,8 \pm 2,3 \mathrm{a}$ \\
\hline Rebrote & $22,7 \pm 1,9 \mathrm{~b}$ & $38,7 \pm 2,6 \mathrm{~b}$ & $25,6 \pm 2,3 \mathrm{a}$ & $70,4 \pm 0,7 \mathrm{~b}$ \\
\hline Floración & $14,7 \pm 1,1 \mathrm{c}$ & $45,8 \pm 3,4 \mathrm{c}$ & $29,1 \pm 2,5 \mathrm{~b}$ & $66,4 \pm 1,7 \mathrm{a}$ \\
\hline
\end{tabular}

Tabla 4.

Media y desvío estándar de los valores de proteína bruta, fibra detergente neutro, fibra detergente ácido y digestibilidad in vitro materia seca de Lycium sp., en distintos periodos vegetativos (2014-2015)

Letras distintas misma columna $(p \leq 0,01)$.

En el caso de Schimus spp, en la tabla 5 se observa que tanto para proteína bruta y para fibra detergente neutro se encontró una diferencia estadística significativa $(p \leq 0,01)$ con mejor calidad forrajera durante el Rebrote, reflejado en un mayor nivel de digestibilidad in vitro materia seca; mientras que los valores de fibra detergente ácido y digestibilidad de materia seca fueron similares entre los periodos de Latencia y Floración, con una diferencia estadística significativa $(p \leq 0,01)$ en la fase de Rebrote.

\section{Tabla 5.}

Media y desvío estándar de los valores de proteína bruta, fibra detergente neutro, fibra detergente ácido y digestibilidad in vitro de materia seca de Schinus spp, en distintos periodos vegetativos (2013-2014)

\begin{tabular}{|c|c|c|c|c|}
\hline SCHINUS & $\% \mathrm{~PB}$ & $\% \mathrm{FDN}$ & $\% \mathrm{FDA}$ & $\% \mathrm{DMO}$ \\
\hline Latencia & $9,1 \pm 0,9 \mathrm{c}$ & $54,5 \pm 1,5^{\mathrm{a}}$ & $34,1 \pm 1,5 \mathrm{~b}$ & $63,2 \pm 1,3 \mathrm{a}$ \\
\hline Rebrote & $19,9 \pm 1,1^{\mathrm{a}}$ & $34,6 \pm 2,2 \mathrm{c}$ & $25,1 \pm 1,5 \mathrm{a}$ & $69,7 \pm 0,9 \mathrm{~b}$ \\
\hline Floración & $14,2 \pm 1,4 \mathrm{~b}$ & $49,2 \pm 1,8 \mathrm{~b}$ & $30,3 \pm 2,5^{\mathrm{a}} \mathrm{b}$ & $64,8 \pm 1,1 \mathrm{a}$ \\
\hline
\end{tabular}

Letras distintas misma columna $(p \leq 0,01)$.
Una situación semejante se encontró con los valores de Schinus spp para el periodo vegetativo 2014-2015, reflejada en una superior calidad nutricional de la especie para el de Rebrote, con una DMO de $70,7 \pm 0,8 \%$, como se observa en la tabla 6. 


\begin{tabular}{|c|c|c|c|c|}
\hline SCHINUS & $\% \mathrm{~PB}$ & $\% \mathrm{FDN}$ & $\% \mathrm{FDA}$ & $\%$ DMO \\
\hline Latencia & $9,6 \pm 0,8 \mathrm{a}$ & $51,6 \pm 1,2 \mathrm{a}$ & $32,4 \pm 2,8 \mathrm{~b}$ & $63,2 \pm 1,9 \mathrm{a}$ \\
\hline Rebrote & $21,1 \pm 0,8 \mathrm{~b}$ & $34,6 \pm 2,2 \mathrm{~b}$ & $70,7 \pm 0,8 \mathrm{~b}$ & $70,4 \pm 0,7 \mathrm{~b}$ \\
\hline Floración & $14,9 \pm 1,3 \mathrm{c}$ & $47,3 \pm 1,6 \mathrm{c}$ & $29,3 \pm 2,2 \mathrm{ab}$ & $65,6 \pm 1,7 \mathrm{a}$ \\
\hline
\end{tabular}

Tabla 6.

Media y desvío estándar de los valores de PB, FDN, FDA y DMO de Schinus Spp, en distintos periodos vegetativos (2014-2015)

Letras distintas misma columna $(p \leq 0,01)$.

En el caso de la evaluación Prosopis sp., la tabla 7 muestra los valores hallados en el lapso 2013-2014, en el que se ve una diferencia estadística significativa $(p \leq 0,01)$ entre los valores de proteína bruta y fibra detergente neutro en los distintos momentos fenológicos, siendo la mayor calidad forrajera la del periodo de Rebrote, con $19,1 \pm 1,3 \%$ de proteína bruta y $70,3 \pm 1,3 \%$ de digestibilidad in vitro materia seca.

\section{Tabla 7.}

Media y desvío estándar de los valores de proteína bruta, fibra detergente neutro, fibra detergente ácido y digestibilidad in vitro materia seca de Prosopis Sp., en distintos periodos vegetativos (2013-2014)

\begin{tabular}{|c|c|c|c|c|}
\hline PROSOPIS & $\% \mathrm{~PB}$ & $\% \mathrm{FDN}$ & $\% \mathrm{FDA}$ & $\% \mathrm{DMO}$ \\
\hline Latencia & $8,3 \pm 0,7 \mathrm{c}$ & $57,4 \pm 2,5 \mathrm{c}$ & $36,9 \pm 2,1 \mathrm{~b}$ & $60,1 \pm 2,1 \mathrm{a}$ \\
\hline Rebrote & $19,1 \pm 1,3^{\mathrm{a}}$ & $35,1 \pm 2,1^{\mathrm{a}}$ & $25,2 \pm 1,3^{\mathrm{a}}$ & $70,3 \pm 1,3 \mathrm{c}$ \\
\hline Floración & $13,6 \pm 0,5 \mathrm{~b}$ & $49,9 \pm 3,1 \mathrm{~b}$ & $31,8 \pm 2,8 \mathrm{~b}$ & $63,6 \pm 1,4 \mathrm{~b}$ \\
\hline
\end{tabular}

Letras distintas misma columna $(p \leq 0,01)$.

Los valores de proteína bruta, fibra detergente neutro, fibra detergente ácido y digestibilidad in vitro materia seca de Prosopis sp., periodo 2014-2015, se presentan en la tabla 8, donde se nota una diferencia estadística significativa $(p \leq 0,01)$ para las variables descritas, según los distintos momentos fenológicos del pastizal natural.

\begin{tabular}{|c|c|c|c|c|}
\hline PROSOPIS & $\% \mathrm{~PB}$ & $\% \mathrm{FDN}$ & $\% \mathrm{FDA}$ & $\%$ DMO \\
\hline Latencia & $8,7 \pm 0,9 \mathrm{a}$ & $54,4 \pm 2,3 \mathrm{~b}$ & $35,1 \pm 2,3 \mathrm{c}$ & $61,7 \pm 1,6 \mathrm{a}$ \\
\hline Rebrote & $19,7 \pm 1,1 \mathrm{c}$ & $35,1 \pm 2,1 \mathrm{a}$ & $25,2 \pm 1,3 \mathrm{a}$ & $71,1 \pm 0,9 \mathrm{~b}$ \\
\hline Floración & $14,4 \pm 0,6 \mathrm{~b}$ & $46,6 \pm 2,7 \mathrm{c}$ & $30,8 \pm 2,6 \mathrm{~b}$ & $64,8 \pm 1,4 \mathrm{c}$ \\
\hline
\end{tabular}

Tabla 8.

Media y desvío estándar de los valores de proteína bruta, fibra detergente neutro, fibra detergente ácido y digestibilidad in vitro materia seca de Prosopis Sp., en distintos periodos vegetativos (2014-2015)

Letras distintas misma columna $(p \leq 0,01)$.

\section{Discusión}

Los resultados encontrados muestran que en la Meseta Central de Mendoza la ingesta caprina en pastizal natural se comportó como lo describieron distintos autores, con una utilización marcada del estrato arbustivo a lo largo de todo el año (Dumont, 1997; Ouédraogo-Koné,
Kaboré-Zoungrana y Ledin, 2006), con tres especies que se destacan: Lucyum sp., Prosopis sp. y Schinus sp, a diferencia de lo descrito por Ramírez (1999), Odo, Omeje y Okwor (2001) y Glassera et al., 2007, quienes destacan que en general no hay preferencia por alguna especie vegetal en particular. 
A su vez, los resultados obtenidos en este trabajo muestran que el aporte en cuanto a la composición de los arbustos forrajeros evaluados fue similar a los descritos por Ku-Vera, 2005 y por Jiménez-Ferrer, López-Carmona, Nahed-Toral, Ochoa-Gaona y De Jong (2008), con una mayor calidad durante el Rebrote y un mínimo en el Reposo vegetativo, valores referenciados en los mayores niveles del porcentaje de proteína bruta, menores valores en los porcentajes de fibra detergente neutro y fibra detergente ácido, que finalmente se traducen en un mayor porcentaje de digestibilidad in vitro materia seca, no encontrando diferencias estadísticas significativas entre los tres arbustos para cada una de variables analizadas y en los distintos momentos fenológicos.

Asimismo, el mayor consumo de arbustos respecto de las gramíneas encontrado en este trabajo en un mismo momento fenológico se debía a la preferencia del ganado caprino por las hojas, que poseen valores nutritivos más elevados en cuanto a cantidad de proteína bruta y menor contenido de pared celular, representado en niveles de fibra detergente neutro y fibra detergente ácido (Sanon, Kabor'e-Zoungrana, Ledin, 2007).

Por otra parte, evaluando cada arbusto en particular, los valores de los porcentajes de proteína bruta fueron superiores y con diferencia estadística significativa $(p \leq 0,01)$ para el periodo de Rebrote en los tres arbustos analizados y fueron mayores los de digestibilidad in vitro materia seca en ese mismo lapso, con una diferencia estadística significativa $(p \leq 0,01)$ en relación con la Floración y al Reposo, tanto en Lycium sp., Schinus sp y Prosopis sp., similar a lo descrito por De la Orden y Quiroga (2006).

En todos los casos, el nivel de proteína bruta resultó dentro de lo encontrado por Benavidez (2006) para una gran cantidad de arbustos forrajeros utilizados por el ganado caprino; los valores que en los tres estados fenológicos cubren los requerimientos proteicos de mantenimiento necesarios para las cabras tipo Criolla regional (NRC, 1981).
Este trabajo permitió definir, en primera instancia, las especies vegetales más utilizadas por el ganado caprino en pastoreo directo diurno del pastizal natural de la Meseta Central de Mendoza, Argentina, y a partir de ello definir la producción forrajera/hectárea de esos arbustos que pasarán a ser especies indicadoras de la condición vegetal de la región y, en segunda instancia, la calidad forrajera de las mismas en diferentes momentos fenológicos y con ello comenzar a diseñar el modelo ideal de ajuste de carga caprina para esa región.

\section{Referencias}

AOAC. (1980). Official Methods of Analices. 13th edition. Washington: Association of Oficial Analytical Chemist.

Barnes, R. y Marten, G. (1980). Recent developments in predicting forage quality. Journal of Animal Science, 48(6), 1554-1561.

Benavidez, J. (2006). Árboles y arbustos forrajeros: una alternativa agroforestal para la ganadería. Conferencia electrónica de la FAO sobre Agroforestería para la producción animal en Latinoamérica. Colombia. Recuperado de http://www.fao.org/livestock/agap/ frg/agrofor1/bnvdes23.pdf

Brinkmann, K., Patzelt, A., Dickhoefer, U., Schlecht, E. y Buerkert, A. (2009). Vegetation patterns and diversity along an altitudinal and a grazing gradient in the Jabal al Akhdar mountain range of northern Oman. Journal of Arid Environments, 73, 1035-1045.

Cabrera, A. (1976). Regiones fitogeográficas argentinas. Enciclopedia argentina de Agricultura y jardinería (pp. 1-85). Buenos Aires: Editorial ACME.

Dayenoff, P. (1998). Contribución al estudio de la sustentabilidad de la ganadería caprina en el Chaco-Arido, Argentina (Tesis Doctoral). Facultad de Veterinaria, Universidad de Murcia. España. 
Dayenoff, P., Bolaño, M., Aguirre, E. y Giovanardi, F. (1999). Efecto del control de la carga caprina sobre la vegetación del Chaco-Arido. Ambiental '99. PRODEA, 1, 87-97.

Dayenoff, P., Macario, J. y Moreno, P. (2013). Caracterización temporal y espacial de la ingesta caprina en la meseta central del sur de Mendoza, Argentina. En Primer Congreso Argentino de Producción Caprina. Gobierno de La Rioja-INTAUMaza-IPAF Cuyo-Universidad Nacional de La Pampa. Ley Caprina.

De la Orden, E. y Quiroga, A. (2006). Evaluación de la calidad y de la oferta de forraje de la hojarasca de una especie arbórea (Celtis tala) del bosque chaqueño. Revista del CIZAS, 7, 30-36.

Dumont, B., (1997). Diet preference of herbivores at pasture. Ann. Zootech., 46,105-116.

El-Gallad, T., Gihad, E., Allam, S. y El-Bedawy, T. (1988). Effect of energy intake and roughage ratio on the lactation of Egyptian Nubian (Zaraibi) goats. Small Ruminat Research, 1, 327-341.

Glasser, T., Landau, S., Ungar, D., Perevolotsky, A., Dvash, L., Muklada, H., Kababya, D. y Walker, J. (2012). Foraging selectivity of three goat breeds in a Mediterranean shrubland. Small Ruminant Research, (102), 7-12.

Hall, E., Specht, R. y Early, C. (1964). Regeneration of the vegetation on Koonamore vegetation reserve, 1926-1962. Australian Journal of Botany, $12,205-264$.

Holechek, J. (1982). Sample preparation technique for microhistological analysis. Journal of Range Management, 35, 267-268.
Jiménez-Ferrer, G., López-Carmona, M., Nahed-Toral, J., Ochoa-Gaona, S. y De Jong, B. (2008). Árboles y arbustos forrajeros de la región norte-tzotzil de Chiapas, México. Veterinaria México, 39(2), 199-213.

Ku-Vera, J. (2005). Nutritive value of trees and shrubs for ruminants. En M.R. Mosquera, J. McAdam, A. Rigueiro-Rodríguez (editors), International Silvopastoral and Sustainable Land Management (pp. 83-86). Lugo: CAB Int.

Laycock, W. (1994). Implications of grazing vs no grazing on today's rangelands. En M. Vavra, W. Laycock y D. Pieper (eds.), Ecological implication of hervibories in the West. Denver: Society for Range Management.

López-Trujillo, R. y García Elizondo, R. (1995). Botanical composition and diet quality of gots grazing natural and grass reseed shrublands. Small Ruminant Research,16, 37-47.

NRC. (1981). Nutrient Requirements of Goats: Angora, Dairy and Meat Goats in Temperate and Tropical Countries. Washington. D.C.: National Academy Press.

Odo, B., Omeje, F. y Okwor, J. (2001). Forage species availability, food preference and grazing behavior of goats in southeastern Nigeria. Small Ruminant Research, 42, 163-168.

Omphile, U., Aganga, A. y Malamba, B. (2003). Diet and forage preference of communally grazed range goats in an Acacia bush savannah in southeast Bostwana. En N. Allsopp, A.R. Palmer, S.J. Milton, Kirkman, G.I.H Kerley, C.R. Hurt y C.J. Brown (eds.), Proceedings of the VIIth International Rangeland Congress (pp. 13011305). Durban, South Africa. 


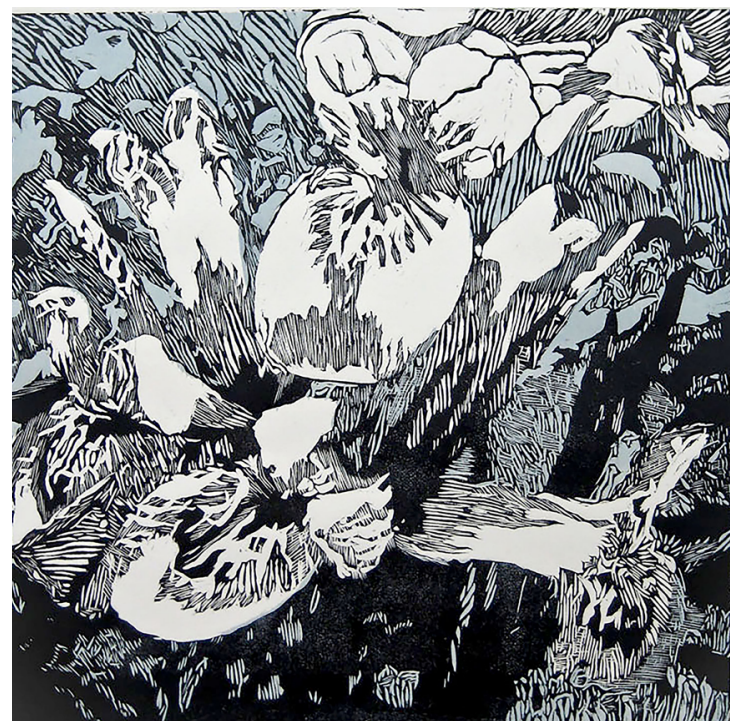

Eva Macías,

"Suspiro",

xilografía,

$60 \times 60 \mathrm{~cm}$,

2015.

Ouédraogo-Koné, S., Kaboré-Zoungrana, C. y Ledin, I. (2006). Behaviour of goats, sheep and cattle on natural pasture in the sub-humid zone of West Africa. Livestock Science, 105, 244-252.

Pande, R., Kemp, P. y Hodgson, J. (2002). Preference of goats and sheep for browse species under field conditions. New Zealand Journal of Agricultural Research, (45), 97-102.

Papachristou, T. y Paranastis, V. (1994). Forage value of mediterranean deciduous woody fodder species and it implication to management of silvo-pastoril system for goats. Agroforestry Systems, (27), 269-282.

Radclife, J. (1985). Grazing management of goats and sheep for gorse control. New Zealand Journal of Experimental Agriculture, 13, 181-190.

Ramírez, R. (1999). Feed resources and feeding techniques of small ruminants under extensive management conditions. Small Ruminant Research, 34, 215-230.

Sanon, H., Kabor'e-Zoungrana, C. y Ledin, I. (2007). Behaviour of goats, sheep and cattle and their selection of browse species on natural pasture in a Sahelian area. Small Ruminant Research, (67), 64-74.

Scarnecchia, D. (1994). A view point. Using multiple variables as indicators in grazing research and management. Journal of Range Management, 47, 107-111.

Severson, K. y Debano, L. (1991). Influence of Spanish goats on vegetation and soil in Arizona chaparral. Journal of range management, 44, 111-117.

Siebert, B., Newman, D. y Nelson, D. (1968). The chemical composition of some arid zone pasture species. Tropical Grassland, 2, 31-40.

Taylor, C., Kothmann, M., Merrill, L, y Elledge, D. (1980). Diet selection by cattle under high, low frequency, short duration and grazing systems. J. Range Management, 33, 428-434.

Van Soest, P., Robertson, B. y Lewis, A. (1991). Methods for dietary fiber, neutral detergent fiber, and non-starch polysaccharides in relation to animal nutrition. Journal of Dairy Science, (10), 3583-3597. 